BNL-112451-2016-JA

\title{
Interface superconductivity in cuprates defies Fermi-Liquid description
}

\author{
Z. Radovic \\ Submitted to Journal of Superconductivity and Novel Magnetism.
}

August 2016

\section{Condensed Matter Physics and Material Science Department Brookhaven National Laboratory}

\section{U.S. Department of Energy USDOE Office of Science (SC), Basic Energy Sciences (BES) (SC-22)}

\footnotetext{
Notice: This manuscript has been authored by employees of Brookhaven Science Associates, LLC under Contract No. DE-SC0012704 with the U.S. Department of Energy. The publisher by accepting the manuscript for publication acknowledges that the United States Government retains a non-exclusive, paid-up, irrevocable, world-wide license to publish or reproduce the published form of this manuscript, or allow others to do so, for United States Government purposes.
} 


\section{DISCLAIMER}

This report was prepared as an account of work sponsored by an agency of the United States Government. Neither the United States Government nor any agency thereof, nor any of their employees, nor any of their contractors, subcontractors, or their employees, makes any warranty, express or implied, or assumes any legal liability or responsibility for the accuracy, completeness, or any third party's use or the results of such use of any information, apparatus, product, or process disclosed, or represents that its use would not infringe privately owned rights. Reference herein to any specific commercial product, process, or service by trade name, trademark, manufacturer, or otherwise, does not necessarily constitute or imply its endorsement, recommendation, or favoring by the United States Government or any agency thereof or its contractors or subcontractors. The views and opinions of authors expressed herein do not necessarily state or reflect those of the United States Government or any agency thereof. 


\title{
Interface superconductivity in cuprates defies Fermi-Liquid description
}

Zoran Radović $^{1}$, Mihajlo Vanević ${ }^{1}$, Jie Wu${ }^{2}$, Anthony T. Bollinger ${ }^{2}$ and Ivan Božović I $^{2,3}$

${ }^{1}$ Department of Physics, University of Belgrade, Studentski trg 12, 11158 Belgrade, Serbia

${ }^{2}$ Condensed Matter Physics and Materials Science Division, Brookhaven National Laboratory, Upton, New York 11973-5000, USA

${ }^{3}$ Applied Physics Department, Yale University, New Haven CT 06520, USA

\section{Corresponding Author:}

Ivan Božović

Phone: $\quad$ (631) 344-4973

Fax: $\quad$ (631) 344-4071

E-mail:_bozovic@bnl.gov

\begin{abstract}
$\mathrm{La}_{2-\mathrm{x}} \mathrm{Sr}_{\mathrm{x}} \mathrm{CuO}_{4} / \mathrm{La}_{2} \mathrm{CuO}_{4}$ bilayers show interface superconductivity that originates from accumulation and depletion of mobile charge carriers across the interface. Surprisingly, the doping level can be varied broadly (within the interval $0.15<x<0.47$ ) without affecting the transition temperature, which stays essentially constant and equal to that in optimally doped material, $T_{c} \approx 40$ $\mathrm{K}$. We argue that this finding implies that doping up to the optimum level does not shift the chemical potential, unlike in ordinary Fermi liquids. We discuss possible physical scenarios that can give doping-independent chemical potential in the pseudogap regime: electronic phase separation, formation of charge-density waves, strong Coulomb interactions, or self-trapping of mobile charge carriers.
\end{abstract}

\section{Keywords}

high-temperature superconductors | interface superconductivity | chemical potential | Fermi liquid 


\section{Introduction}

High-temperature interface superconductivity (HT-IS) was probably first observed by J. Eckstein and I. Bozovic (then at Varian Research Center in Palo Alto, California), in experiments ${ }^{1}$ with heterostructures made out of various phases from the $\mathrm{Bi}_{2} \mathrm{Sr}_{2} \mathrm{Ca}_{n-1} \mathrm{Cu}_{n} \mathrm{O}_{2 n+2}$ family of compounds. Thin films were synthesized using atomic-layer-by-layer molecular beam epitaxy (ALL-MBE), and HT-IS with $T_{c}=75 \mathrm{~K}$ was induced ${ }^{2}$ in $\mathrm{Bi}-2278$ when it was placed in contact with overdoped Bi-2201. Subsequently, HT-IS was also observed in heterostructures based on $\mathrm{La}_{2-\mathrm{x}} \mathrm{Sr}_{\mathrm{x}} \mathrm{CuO}_{4}$ $(\mathrm{LSCO})^{3}$.

The interest in this topic surged dramatically after discovery of HT-IS in bilayers in which neither of the constituent materials is superconducting in isolation, such as $\mathrm{La}_{2} \mathrm{CuO}_{4}$ (which is an insulator) and $\mathrm{La}_{1.55} \mathrm{Sr}_{0.45} \mathrm{CuO}_{4}$ (which is a non-superconducting metal) ${ }^{4}$. This phenomenon is very robust; by now it has been observed in hundreds of such bilayers, without an exception, and the samples are stable on a scale of (at least) 10 years. The digital ALL-MBE synthesis ${ }^{5}$ made it possible to perform incisive experiments (“Zn- $\delta$-doping tomography”) which proved that HT-IS is confined to a single $\mathrm{CuO}_{2}$ layer ${ }^{6}$, the location of which can be unambiguously identified. Another series of experiments, using advanced characterization techniques such as atomicresolution electron-energy loss spectroscopy (HR-EELS) by means of scanning transmission electron microscope (STEM), time-of-flight ion scattering and recoil spectroscopy (TOFISARS), and coherent Bragg rod analysis (COBRA) phase-retrieval using synchrotron X-ray radiation, established that there was very little cation inter-diffusion across the nominal geometric interface $^{4,6,7}$. Basically, under the standard ALL-MBE growth conditions, Sr ions only migrate within a single LSCO layer (0.66 nm thick, one-half the crystallographic unit cell) ${ }^{8}$. Complementary experiments using soft resonant X-ray scattering technique (SRXRS) ${ }^{9}$ showed that HT-IS is primarily caused by the redistribution of the doped holes across the interface, as a consequence of the difference in the chemical potential $(\mu)$ of the two layers doped to a different level.

The next key question was how $T_{c}$ of such LSCO/LCO bilayers depends on the doping level in LSCO. This was important as a test of various models, and in particular of the prediction that $T_{C}$ could be significantly enhanced by proximity effects between an underdoped and overdoped LSCO, thus optimizing both the superfluid phase stiffness and the strength of pairing interac-

tion $^{10}$. To address this question, we have performed a systematic study ${ }^{11}$ that encompassed more 
than 800 LSCO/LCO bilayer samples, with the doping level in LSCO varied across a broad interval $0.15<x<0.47$, in exquisitely fine steps ( $\delta x<0.0005)$. The main result ${ }^{11}$ was that $T_{c}$ of the bilayers was essentially independent on the doping level in LSCO, see Figure 1 (left panel). In what follows, we offer an explanation of this surprising result.

\section{Theoretical model and results}

To calculate the charge profile, we model phenomenologically the electrostatic properties along the $c$-axis of LSCO/LCO bilayer samples ${ }^{11}$. Far from the interface, on the metallic side the mobile holes with density $p$ per $\mathrm{CuO}_{2}$ plane are in equilibrium with ionized dopants of density $x$, $p=x$. Near the interface, the difference between chemical potentials in overdoped and undoped layers leads to redistribution of the charge carriers. The induced electrostatic potential $\phi$ is related to the excess charge density by the Poisson equation

$$
\frac{d^{2} \phi}{d z^{2}}=-\frac{2 e(p-x)}{\epsilon_{0} \epsilon_{r} a^{2} c},
$$

where $a=0.38 \mathrm{~nm}$ and $c=1.32 \mathrm{~nm}$ are in-plane and out-of-plane lattice constants $(c / 2$ is the distance between $\mathrm{CuO}_{2}$ planes), and $\epsilon_{r}$ is the effective dielectric permittivity along $c$ axis. We take $\epsilon_{r}=50$ as representative of the values measured experimentally ${ }^{12}$. We choose $z=0$ at the geometrical interface between overdoped (metallic) $z<0$, and undoped (insulating) $z>0$ layers.

In equilibrium, the electrochemical potential is constant along the sample,

$$
-e \phi+\mu=\text { const. }
$$

The $\mu(p)$ dependence is modeled based on angle-resolved photoemission spectroscopy (ARPES) $\operatorname{data}^{13}: \mu(p)=0$ for $p<p_{0}$, and $\mu(p)=-1.5\left(p-p_{0}\right) \mathrm{eV}$ for $p>p_{0}$, (see Figure 2 ), where $p_{0}=0.16$ corresponds to the maximum $T_{c}$. Note that this dependence was also supported by the absence of charge transfer between LCO and optimally doped LSCO in heterostructures and superlattices grown by ALL-MBE ${ }^{14}$. Simple analytical solutions are:

$$
\begin{gathered}
p(z)=x\left(1-\frac{x^{2}-p_{0}^{2}}{2 x^{2}} e^{\kappa z}\right) \text { for } z<0, \\
p(z)=p_{0} \cosh \left[\kappa\left(z-z_{0}\right)\right] \text { for } 0 \leq z \leq z_{0},
\end{gathered}
$$


and $p=0$ for $z>z_{0}$. Here, $\kappa^{2}=2 e^{2} /\left(\epsilon_{0} \epsilon_{r} a^{2} c \times 1.5 \mathrm{eV}\right)$ and $z_{0}=\kappa^{-1} \ln \left(x / p_{0}\right)$. Although we solve a continuum model, the resulting $p(z)$ profile is assigned to $N= \pm 1, \pm 2 \quad \mathrm{CuO}_{2}$ planes at discrete values of $z= \pm c / 4, \pm 3 c / 4$, and related to $T_{c}$ by the phenomenological equation $T_{c}(p)=$ $4 \times 10^{3}(p-0.06)(0.26-p)[\mathrm{K}]$. Analytical solutions are depicted in Figure 3 (left panel) for $x=0.45$, 0.33 , and 0.26 . It can be seen that interface superconductivity occurs at the maximum $T_{c}$ in the first or second $\mathrm{CuO}_{2}$ plane independent of the Sr concentration $x$ in the overdoped layer, in good agreement with the experimental data, see Figure 1. This is a direct consequence of the pinned chemical potential, $\mu(p)=0$ for $p<p_{0}$.

Experimentally detected weak Sr diffusion across the interface does not change the results qualitatively. The nominal Sr profile is taken from the TOF-ISARS data $^{4}$ as an exponential decay from $x$ in the overdoped layer to 0 in the underdoped one: $x(z)=(x / 2) \exp \left(-z / \lambda_{s r}\right)$ for $z>0$, and $x(z)=x\left[1-(1 / 2) \exp \left(z / \lambda_{S r}\right)\right]$ for $z<0$ with $\lambda_{s r}=c / 2$. The equations are solved for $p(z)$ with the boundary conditions $p=x$ when $z \rightarrow-\infty$ and $p=0$ when $z \rightarrow+\infty$ which ensures charge neutrality of the system. We emphasize that for chosen parameters, the charge profile $p(z)$ exponentially approaches $x$ in the overdoped region with the characteristic length $\lambda=0.72 \mathrm{~nm}$.

\section{Discussion}

The big remaining question is why $\mu(p)$ has the 'broken' shape as shown in Figure 2, to begin with. According to ARPES ${ }^{13}$ experiments, in overdoped LSCO the Fermi surface is basically cylindrical and hence the density of states should be doping-independent while the $\mu(p)$ dependence should be linear. Hence, the broken $\mu(p)$ dependence is not expected within a standard Fermi liquid description. Several possible explanations of this non-Fermi liquid behavior have been proposed or can be considered.

One explanation postulates phase separation in real space, in analogy with van der Waals/Maxwell construction, assuming that $\mu(x)$ is constant as long as droplets of hole-rich, optimally-doped phase coexist with and are embedded into an insulating anti-ferromagnetic ma-

trix $^{15,16}$. While this would explain the observed broken $\mu(p)$ and hence the independence of $T_{c}$ on doping in LSCO/LCO bilayers, it clashes with other experimental observations. In optimally 
doped LSCO, the (screened) plasma frequency is $\operatorname{high}^{17}(\sim 0.8 \mathrm{eV})$ and thus the Coulomb interaction between the mobile carries and the ionic lattice would not allow purely electronic phase separation; at the minimum, the lattice would need to distort accordingly. Next, transport measurements do not show a percolation jump from an anti-ferromagnetic insulator to a metal (and hence from $T_{c}=0$ to $T_{c}=40 \mathrm{~K}$ ) but rather a continuous evolution. In principle, one could patch this model to recover the continuous increase of $T_{c}$ by assuming that optimally doped islands are Josephson coupled, with coupling that increases gradually with doping. If this were the case, one would expect to see the telltale signatures of such Josephson contacts - nonlinear $I-V$ characteristics, great sensitivity to microwave radiation and magnetic field, etc. However, none of these are seen; e.g., $H_{c 2}$ is in fact quite high. Moreover, in LSCO films grown by ALL-MBE the superfluid appears to be quite homogeneous ${ }^{18}$. Underdoped $\mathrm{YBa}_{2} \mathrm{Cu}_{3} \mathrm{O}_{6.5}$ also appears to be quite clean (in fact, 'ultra-clean') and homogeneous, based e.g., on quantum (Shubnikov-de Haas and de Haas-van Alphen) oscillations ${ }^{19,20}$ and microwave experiments that show mean free path (of Bogoliubov quasiparticles, at low $T$ ) in 5-10 $\mu \mathrm{m}$ range $^{21}$.

A more likely alternative is "phase separation in k-space", where charge density wave (CDW) forms over a fraction of the Fermi surface ${ }^{22}$, leading to its reconstruction and formation of small arcs (or pockets, as indicated by quantum oscillations). In this way, $\mu(x)$ could likewise stay nearly constant while the pockets evolve upon doping; once the pockets are gone and a single large Fermi surface is established (somewhere near optimal doping), $\mu(x)$ should drop fast and linear in $x$, as observed.

A third option can be envisioned based on the studies of 2D Hubbard model using dynamic mean-field theory (DMFT) calculations ${ }^{23}$ by G. Kotliar and coworkers. They predicted that in a Mott insulator, upon doping mid-gap states form on the account of upper (UHB) and lower (LHB) Hubbard bands. With further doping, these midgap states grow and broaden, eventually merging together with LHB and UHB into a single broad band.

As yet another alternative, midgap states could also arise by self-trapping of mobile charge carriers, i.e., by formation of polarons, or spin-polarons, as indeed proposed by K. A. Mueller, N. Mott, P.-G. De Gennes, G. Deutscher, D. Mihailovic, A. Bussman-Holder, H. Keller, T. Egami, A. Alexandrov, D. Emin, A. Heeger, etc., (see, e.g., Refs. 24-27 and references therein). 
Regrettably, the experimental result that $T_{C}$ is independent on $x$ in LSCO/LCO bilayers only implies that $\mu(p)$ is (nearly) constant up to optimal doping, while it cannot differentiate by itself between the different explanations of the origin of this strange, non-FL behavior. Other, independent experiments are needed for this, and are under consideration.

Looking beyond cuprates, in a very recent tour-de-force experiment, Yamaura and coworkers ${ }^{28}$ have shown that HT-IS with $T_{c} \approx 35 \mathrm{~K}$ can be induced in $\mathrm{Ca}_{0.94} \mathrm{La}_{0.06} \mathrm{Fe}_{2} \mathrm{As}_{2}$ by electron doping, which was achieved by transition-metal (Co, Ni) substitution. The two-dimensional (2D), interfacial nature of superconductivity was verified by measurements of angle-dependent magnetoresistance oscillations. Quite remarkably, the onset $T_{c}$ was found to stay constant for a wide range of doping. Possible extrinsic factors like phase separation, chemical inhomogeneity, and charge-carrier cancelation effects have all been excluded. The broader significance of this discovery is that, together with earlier similar observations in LSCO/LCO heterostructures, it may indicate that the inferred non-FL behavior may be common to both cuprates and pnictides, perhaps implying deeper connections and similar nature of superconductivity in these materials.

\section{References:}

1. Eckstein, J. N., Bozovic, I., Schlom, D. G., Harris Jr., J. S.: Growth of superconducting $\mathrm{Bi}_{2} \mathrm{Sr}_{2} \mathrm{Ca}_{\mathrm{n}}$ ${ }_{1} \mathrm{Cu}_{\mathrm{n}} \mathrm{O}_{\mathrm{x}}$ thin films by atomically layered epitaxy. J. Cryst. Growth 111, 973-7 (1991).

2. Bozovic, I., Eckstein, J. N., Virshup, G. F.: Superconducting Oxide Multilayers and Superlattices: Physics, Chemistry, and Nano-engineering. Physica C 235-240, 178-181 (1994).

3. Bozovic, I., Logvenov, G., Belca, I., Narimbetov, B., Sveklo, I.: Epitaxial strain and superconductivity in $\mathrm{La}_{2-\mathrm{x}} \mathrm{Sr}_{\mathrm{x}} \mathrm{CuO}_{4}$ thin films. Phys. Rev. Lett. 89, 107001 (2002).

4. Gozar, A., Logvenov, G., Fitting Kourkoutis, L., Bollinger, A. T., Giannuzzi, L. A., Muller, D. A., Božović, I.: Interface superconductivity between a metal and a Mott insulator. Nature 455, 782 (2008).

5. Bozovic, I.: Atomic-layer engineering of superconducting oxides: Yesterday, today, tomorrow. IEEE Trans. Appl. Supercond. 11, 2686-2695 (2001).

6. Logvenov, G., Gozar, A., Bozovic, I.: High-Temperature Superconductivity in a Single CopperOxygen Plane. Science 326, 699 (2009).

7. Bollinger, A. T., Dubuis, G., Yoon, J., Pavuna, D., Misewich, J., Bozovic, I.: Superconductor-insulator transition in $\mathrm{La}_{2-\mathrm{x}} \mathrm{Sr}_{\mathrm{x}} \mathrm{CuO}_{4}$ at the pair quantum resistance. Nature 472, 458 (2011).

8. Yacoby, Y., Zhou, H., Pindak, R., Božović, I.: Atomic-layer synthesis and imaging uncover broken inversion symmetry in $\mathrm{La}_{2-x} \mathrm{Sr}_{x} \mathrm{CuO}_{4}$ films. Phys. Rev. B 87, 014108 (2013).

9. Smadici, S., Lee, J. C. T., Wang, S., Abbamonte, P., Logvenov, G., Gozar, A., Deville Cavellin, C., Bozovic, I.: Superconducting transition at $38 \mathrm{~K}$ in insulating-overdoped $\mathrm{La}_{2} \mathrm{CuO}_{4}-\mathrm{La}_{1.64} \mathrm{Sr}_{0.36} \mathrm{CuO}_{4}$ 
superlattices: evidence for interface electronic redistribution from resonant soft X-ray scattering. Phys. Rev. Lett. 102, 107004 (2009).

10. Kivelson, S.A.: Making high- $\mathrm{T}_{\mathrm{C}}$ higher: a theoretical proposal. Physica B 318, 61 (2002).

11. Wu, J., Pelleg, O., Logvenov, G., Bollinger, A. T., Sun, Y., Boebinger, G. S., Vanević, M., Radović, Z., Božović, I.: Anomalous independence of interface superconductivity on carrier density. Nature Materials 12, 877 (2013).

12. Chen, C. Y., et al.: Frequency and magnetic-field dependence of the dielectric constant and conductivity of $\mathrm{La}_{2} \mathrm{CuO}_{4+\mathrm{y}}$. Phys. Rev. B 43, 392-401 (1991).

13. Ino, A., Mizokawa, T., Fujimori, A., Tamasaku, K., Eisaki, H., Uchida, S., Kimura, T., Sasagawa,T., Kishio, K.: Chemical Potential Shift in Overdoped and Underdoped $\mathrm{La}_{2-\mathrm{x}} \mathrm{Sr}_{\mathrm{x}} \mathrm{CuO}_{4}$. Phys. Rev. Lett. 79, 2101 (1997).

14. Bozovic, I., Logvenov, G., Verhoeven, M. A. J., Caputo, P., Goldobin, E., Geballe, T. H.: No mixing of superconductivity and antiferromagnetism in a high-temperature superconductor. Nature $\mathbf{4 2 2}$, 873 (2003).

15. van der Marel, D.: Interface superconductivity: Pinning the critical temperature. Nature Mater.12, 875-876 (2013).

16. Misawa, T., Imada, M.: Origin of high- $\mathrm{T}_{\mathrm{c}}$ superconductivity in doped Hubbard models and their extensions: Roles of uniform charge fluctuations. Phys. Rev. B 90, 115137 (2014).

17. Kim, J. H., Bozovic, I., Eom, C. B., Geballe, T. H., Harris Jr., J. S.: Study of optical plasmons in $\mathrm{La}_{2-\mathrm{x}} \mathrm{Sr}_{\mathrm{x}} \mathrm{Cu}_{2} \mathrm{O}_{4}$. Physica C 174, 435-9 (1991).

18. Božović, I., He, X., Wu, J., Bollinger, A. T.: Dependence of critical temperature in overdoped copper oxides on superfluid density. Nature (2016) in press.

19. Doiron-Leyraud, N., Proust, C., LeBoeuf, D., Levallois, J., Bonnemaison, J. B., Liang, R., Bonn, D. A., Hardy, W. N., Taillefer, L.: Quantum oscillations and the Fermi surface in an underdoped high$\mathrm{T}_{\mathrm{c}}$ superconductor. Nature 447, 565 (2007).

20. Jaudet, C. et al.: De Haas-van Alphen Oscillations in the Underdoped High-Temperature Superconductor $\mathrm{YBa}_{2} \mathrm{Cu}_{3} \mathrm{O}_{6.5}$. Phys. Rev. Lett. 100, 187005 (2008).

21. Hosseini, A. et al.: Microwave spectroscopy of thermally excited quasiparticles in $\mathrm{YBa}_{2} \mathrm{Cu}_{3} \mathrm{O}_{6.99}$. Phys. Rev. B 60, 1349-1359 (1999).

22. Blackburn, E.: X-ray diffraction studies of charge density waves in cuprate superconductors: A brief review. Physica B 460, 132-135 (2015).

23. Kotliar, G., Vollhardt, D.: Strongly correlated materials: Insights from dynamical mean-field theory. Phys. Today 57, 53-59 (2004).

24. Zhao, G. M., Hunt, M. B., Keller, H., Muller, K. A.: Evidence for polaronic supercarriers in the copper oxide superconductors $\mathrm{La}_{2-\mathrm{x}} \mathrm{Sr}_{\mathrm{x}} \mathrm{CuO}_{4}$. Nature 385, 236-239 (1997).

25. Bishop, A. R., Mihailovic, D., de Leon, J. M.:Signatures of mesoscopicJahn-Teller polaron inhomogeneities in high-temperature superconductors. J. Phys. Cond. Mat. 15, L169-L175 ( 2003).

26. Rosch, O., Gunnarsson, O., Zhou, X. J., Yoshida, T., Sasagawa, T., Fujimori, A., Hussain, Z., Shen, Z.-X., Uchida, S.: Polaronic behavior of undoped high- $\mathrm{T}_{\mathrm{c}}$ cuprate superconductors from angleresolved photoemission spectra. Phys. Rev. Lett. 95, 227002 (2005).

27. Bussmann-Holder, A., Keller, H.: Polaron formation as origin of unconventional isotope effects in cuprate superconductors. Europhys. J. B. 44, 487-490 (2005). 
28. W. Zhou, X. Z. Xing, H. J. Zhao, Z. X. Shi and K. Yamaura. Anomalous carrier density independent superconductivity in iron pnictides. ArXiv:1604.02839v1 (11 April 2016).

Acknowledgements. The experimental work was done at BNL and was supported by the U.S. Department of Energy, Basic Energy Sciences, Materials Sciences and Engineering Division. 

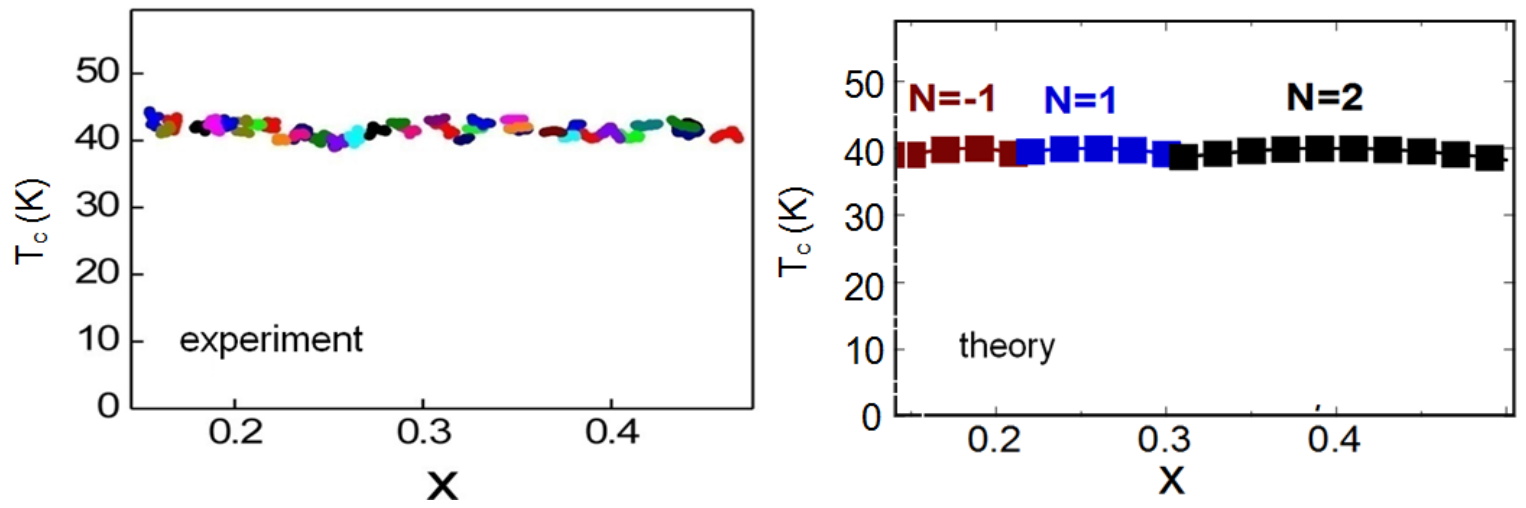

Figure 1. The doping dependence of $T_{C}$ in metallic $\mathrm{La}_{2-\mathrm{x}} \mathrm{Sr}_{\mathrm{x}} \mathrm{CuO}_{4} /$ insulating $\mathrm{La}_{2} \mathrm{CuO}_{4}$ bilayer films: experimental data (left panel) and theoretical results (right panel). Superconductivity occurs in the $\mathrm{N}$-th $\mathrm{CuO}_{2}$ plane away from the interface (on the LCO side). 


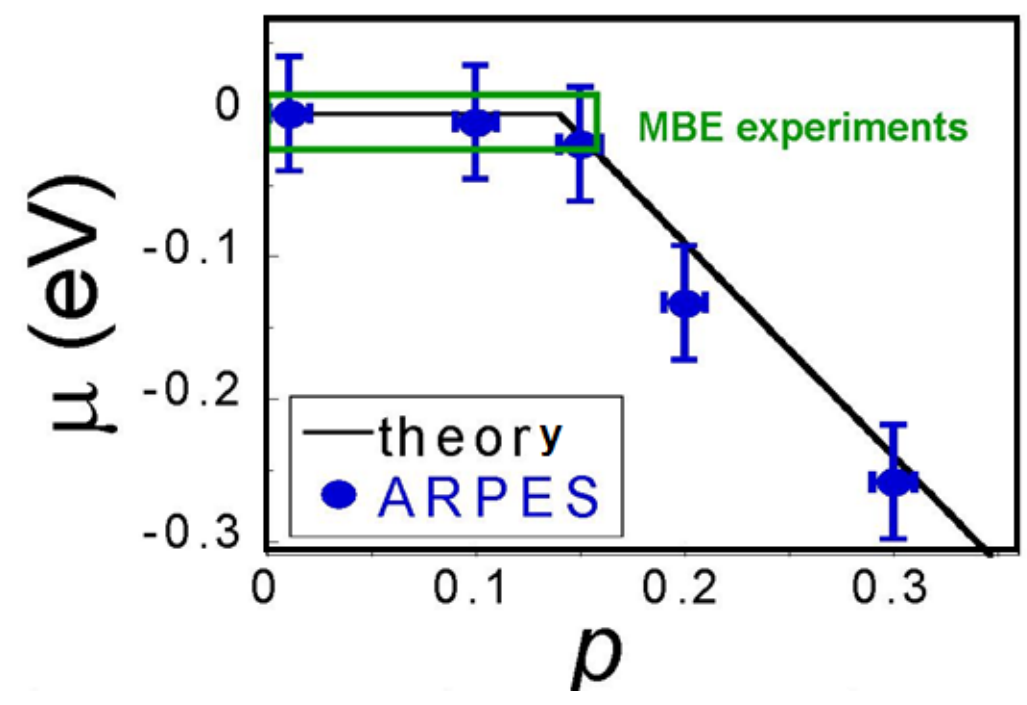

Figure 2. The chemical potential as a function of the hole density $p$ is constant up to the optimal doping (see Refs. [13], [14]). For theoretical modeling we use a piecewise-linear dependence shown by the solid line. 

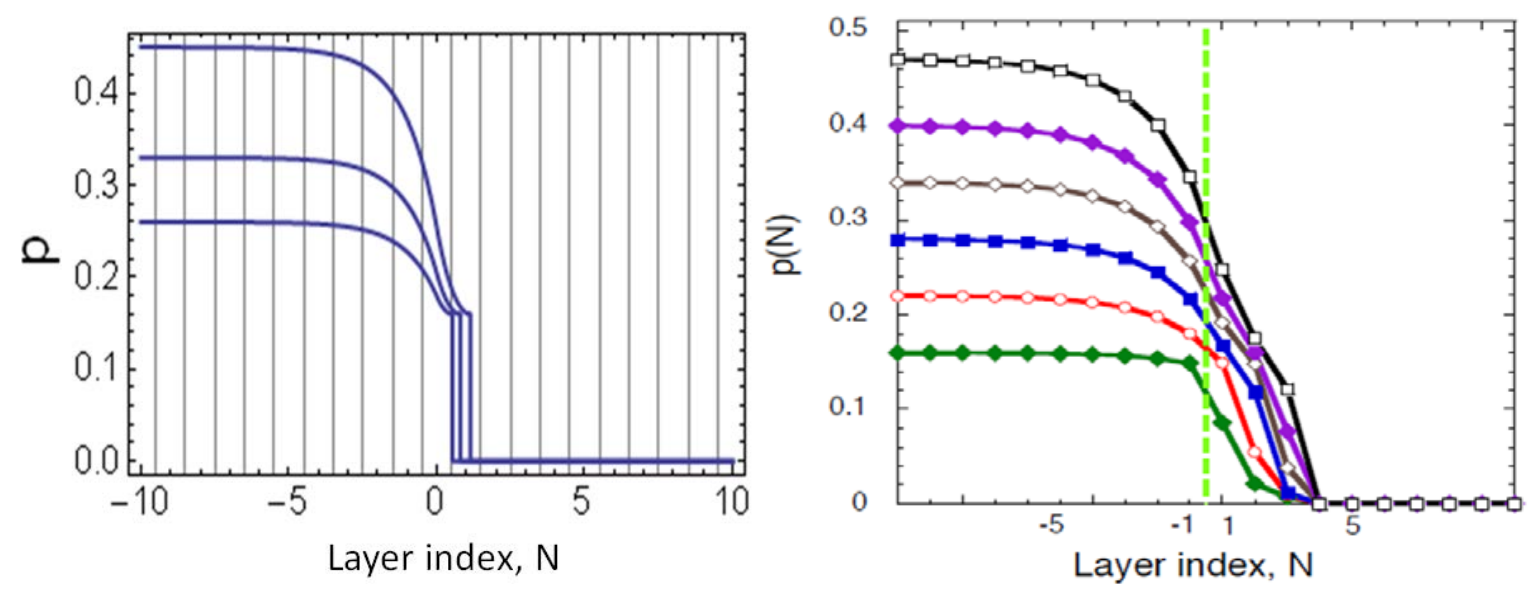

Figure 3. The equilibrium hole density $p$ at $\mathrm{Nth} \mathrm{CuO}_{2}$ plane in a LSCO/LCO bilayer, calculated analytically (left panel) and numerically taking into account $\mathrm{Sr}$ diffusion (right panel). Curves correspond to different doping levels $x$ ( $x=p$ in the metallic layer far from the interface). The dependence of the chemical potential on the hole density is assumed to be the same as the one shown in Figure 2. 